\title{
Modèles de curricula et politiques curriculaires en Italie
}

Curricula models and policies in Italy

Modelos de los planes de estudio y políticas curriculares en Italia

Arduino Salatin

Traducteur : Roger-François Gauthier et Luisa Lombardi

\section{(2) OpenEdition}

Journals

Édition électronique

URL : https://journals.openedition.org/ries/1043

DOI : $10.4000 /$ ries. 1043

ISSN : 2261-4265

Éditeur

France Education international

Édition imprimée

Date de publication : 1 avril 2011

Pagination : 41-50

ISBN : $978-2854205916$

ISSN : 1254-4590

Référence électronique

Arduino Salatin, "Modèles de curricula et politiques curriculaires en Italie », Revue internationale

d'éducation de Sèvres [En ligne], 56 | avril 2011, mis en ligne le 01 avril 2014, consulté le 04 mai 2021.

URL : http://journals.openedition.org/ries/1043; DOI : https://doi.org/10.4000/ries.1043 


\section{Modèles de curricula et politiques curriculaires en Italie*}

\section{Arduino Salatin}

\section{LA NOTION DE CURRICULUM ET SON ÉVOLUTION}

On trouve au niveau international de très nombreuses définitions du concept de curriculum ainsi que de ses mises en pratique ${ }^{1}$. La recherche qui s'est développée durant les dernières décennies, en Italie comme ailleurs, a permis de comprendre que la réflexion sur le curriculum est une activité complexe, qui touche à tout un ensemble d'éléments que l'on retrouve dès qu'il est question d'éducation : finalités générales, objectifs à faire atteindre par les élèves (en termes de tâches à accomplir comme de niveaux de performances), contenus d'enseignement, méthodes pédagogiques et modes d'organisation des études, modalités d'évaluation des activités d'enseignement comme des expériences d'apprentissages.

Le curriculum est défini selon les cas soit :

- comme un ensemble de contenus qui se présentent en termes de savoirs ou encore de disciplines; d'ordinaire, cette acception renvoie à la notion de "syllabus", comprise comme un liste de contenus à apprendre, qui doivent être transmis aux élèves dans le cadre d'un cursus, en liaison étroite avec un ensemble d'exercices canoniques et de tests sommatifs;

- comme une "programmation de l'enseignement ", avec aussi bien ce qui concerne l'organisation des contenus à enseigner que ce qui peut garantir l'atteinte des objectifs, en tentant d'optimiser les dépenses et les formes d'organisation (horaires, répartition des enseignements, etc.) ;

- comme un ensemble d'objectifs d'apprentissage et/ou de performances ; cette approche se centre sur l'ensemble des capacités, savoirs et compétences qu'un élève doit acquérir à l'issue d'un cursus : elle déplace le centre de gravité de l'éducation de l'enseignant à l'élève, en tant qu'il est le véritable bénéficiaire ;

\footnotetext{
* Article traduit par Roger-François Gauthier et Luisa Lombardi.

1. Aucun doute sur le fait que le mot de « curriculum » est une importation anglo-saxonne. Il est moins vrai, même si on le lit souvent, que le concept renvoie à un contexte d'autonomie d'établissement. Le concept apparaît au début du XXe siècle aux États-Unis comme outil qui permet de contrôler et de documenter de façon cohérente le déroulement des actions pédagogiques et de formation (F. Bobbit, 1918).
} 
- ou encore comme ce que l'élève acquiert en termes d'instruction et de formation aussi bien à l'intérieur qu'à l'extérieur de l'école, sous la guidance des enseignants. Dans cette perspective, le curriculum est constitué de l'ensemble des expériences d'apprentissage d'un individu qui résultent de sa scolarisation : l'apprentissage purement scolaire ne constitue pas la seule expérience d'apprentissage qui ait du sens, mais elle est une occasion pour chacun de réfléchir et de se socialiser. Ici, l'accent est mis sur l'élève en tant que sujet de ses apprentissages, actif et motivé, et aussi en position d'évaluer les résultats du curriculum pour sa propre formation.

Au cours des trente dernières années, en Italie, à la suite du débat sur l'éducation, l'attention s'est progressivement déplacée (même dans les textes officiels) de la notion de "programme» (émanant du ministère) à celle de «curriculum » et ultérieurement à celle de "plan d'études personnalisé $~^{2}$ : on est passé de quelque chose de défini d'en haut à l'idée d'un parcours de formation complexe à construire avec les élèves eux-mêmes. Ce passage entraîne l'abandon d'une conception du curriculum centrée sur un certain nombre de connaissances considérées comme générales et valables pour tous à une conception par laquelle il s'agit de favoriser le développement de " compétences » (culturelles, professionnelles, personnelles, etc.).

Le programme émanant du ministère définit « ce qui doit être enseigné » ${ }^{3}$, ce qui, aussi bien dans la définition des objectifs que dans la répartition des sujets et des contenus, s'impose comme la référence unique pour l'ensemble du territoire national comme pour tous les enseignements d'un niveau déterminé d'instruction. Quant au curriculum, en revanche, offrant à chaque élève la possibilité de choisir son itinéraire, son rythme et son but, il définit «ce qui doit être appris » 4 .

Le curriculum devient alors le système organisé des offres de formation et des modalités d'intervention pédagogiques proposées par un établissement, un groupe de classes ou même une classe particulière (en précisant l'utilisation du temps, les méthodes, les contenus et, pour certains, les objectifs en termes de savoirs, de compétences et de savoir être à faire atteindre aux élèves). Selon une telle orientation, qui s'est généralisée en Italie à la fin du siècle précédent, le curriculum se centre sur la compétence définie comme une maîtrise consciente de connaissances, de capacités, de savoir-faire personnels, sociaux et méthodologiques, mis en œuvre de façon autonome et responsable, à travers les savoirs qui se sont constitués dans le cadre des apprentissages.

2. "Piano di studio » dans le texte italien, est expliqué plus bas : le mot à mot français " plan d'études », évoquant précisément une réalité uniforme, ne conviendrait pas (NdT).

3. L'opposition, dans le texte italien, entre " piano di insegnamento » et « piano di apprendimento » ne nous semble pas pouvoir être rendue avec des substantifs français « plan », ou « programme » (NdT).

4. Une telle évolution a été rendue possible aussi par la rencontre entre la tradition pédagogique italienne et la recherche anglo-américaine de la deuxième moitié des années 1960. Prévalut, dans un premier temps, une sorte d'adaptation passive à la terminologie anglaise, et la notion de curriculum était utilisée pour dire " plan d'études », ou « programme ministériel personnalisé librement par l'enseignant ». 
L'école obligatoire ${ }^{5}$ est censée élaborer son modèle de curriculum en liaison avec son objectif spécifique : le développement des apprentissages, désormais devenu un des droits fondamentaux. L'approche didactique s'en trouve modifiée : on passe du simple au complexe, selon un mouvement en spirale qui garantit la progressivité de l'apprentissage en termes de compétences. Structure curriculaire et processus d'apprentissage sont fortement reliés et complémentaires, ce qui conduit à se poser la question du rapport entre les changements qu'exige le développement de l'élève et la stabilité des disciplines.

L'école secondaire supérieure se réfère à un "profil culturel, professionnel et personel » propre à chaque filière d'études, avec les résultats, en termes d'apprentissage $^{6}$, qui lui sont propres, tandis la formation professionnelle se réfère à des "profils professionnels", correspondant chacun à une qualification ou à un diplôme, profils construits comme de véritables "référentiels de compétences » spécifiques.

\section{ÉLABORATION ET RÉGULATION DES CURRICULA}

La tradition italienne centralisatrice, fondée sur des lois, des décrets, des orientations et des programmes émanant du ministère, a conditionné l'élaboration même la plus récente des curricula et limité de fait l'implication des personnels.

Au tournant du siècle s'est dessinée une nouvelle phase caractérisée par :

- la mise en place de la réforme sur l'autonomie en matière scolaire (loi $n^{\circ} 59$ de 1997, art. 21), avec son décret d'application ( ${ }^{\circ}$ 275/1999) : le centralisme traditionnel des programmes est atténué et ils sont revus dans le sens de la mise au point de curricula plus plastiques;

- des essais de mise en place de la réforme de l'école (2000 et 2003), qui a concerné l'ensemble du système éducatif, les contenus d'enseignement aussi bien que les méthodes et l'organisation pédagogique.

C'est dans le Regolamento dell'autonomia (1999) qu'est formalisé juridiquement le passage "de l'école des programmes scolaires à celle des curricula ». L'article 8 donne en effet aux écoles la compétence de bâtir le curriculum, tout en garantissant l'existence d'un système éducatif national avec un ministère chargé de définir les « objectifs spécifiques des apprentissages liés aux compétences des élèves ».

5. En Italie, depuis 2007, l'obligation scolaire va jusqu'à 16 ans; elle est constituée de dix ans (cinq années de primaire, trois de secondaire inférieur, et deux correspondant aux premières années du secondaire supérieur ou de la formation professionnelle, confiée aux régions).

6. L'école secondaire supérieure a été réformée en 2010 ; elle est constituée d'une façon arrêtée par l'État de parcours de cinq ans : lycée (avec six filières), voie technologique (onze filières) et voie professionnelle (six filières). À l'issue de ces parcours, l'élève peut passer un examen débouchant sur un diplôme d'État qui donne accès à l'Université ou à l'enseignement technique supérieur. À côté de ce système, on trouve le système régional de formation professionnelle, avec des cursus de trois à quatre années (toujours après 14 ans), qui donnent accès à une qualification ou à un diplôme professionnel reconnu au niveau national. 
Le curriculum se compose :

- d'une proportion définie par le ministère au plan national ;

- d'une proportion qui est de la compétence des établissements, correspondant à $20 \%$ de l'horaire, qui tient compte de la spécificité de chaque école et des besoins locaux.

Le Regolamento prévoit en outre que chaque établissement produit annuellement son Plan de l'offre de formation (POF). Le POF est établi par le conseil de professeurs de l'établissement et constitue le document sur lequel se fonde le projet de l'établissement et son identité culturelle.

Le cœur pédagogique du POF est le curriculum de l'établissement, qui est produit par l'ensemble des professionnels (chef d'établissement et enseignants), dans le respect des orientations et des limites posées par les textes nationaux : son élaboration est l'occasion de mesurer concrètement la capacité de chaque école à bâtir son projet.

Une conséquence de l'introduction de l'autonomie est que le lieu de la décision se déplace, au moins en partie, du centre vers chaque établissement: pour ce motif, le curriculum s'affirme comme l'instrument principal de son projet pédagogique.

Pour respecter et valoriser cette autonomie des établissements, les Indications nationales constituent le cadre des référence des choix qui sont de la compétence de ces établissements : en combinant compétence nationale sur le curriculum pour la partie nationale et compétence d'établissement, on garantit l'existence du système éducatif et on valorise un pluralisme culturel et territorial, dans le respect des finalités propres de l'école obligatoire et du secondaire supérieur ${ }^{7}$. Le curriculum que chaque établissement élabore constitue une mosaïque dont le dessin d'ensemble est commun et que chaque communauté professionnelle locale est appelée à assumer et à contextualiser, en tenant compte des besoins de développement des élèves, des attentes de la société, des ressources disponibles aussi bien à l'intérieur de l'école qu'au niveau local. Pour cela les établissements doivent innover au plan didactique et pédagogique, gérer la formation de leurs personnels, produire et diffuser des outils pédagogiques, constituer les réseaux d'établissements susceptibles de favoriser les échanges d'informations, d'expériences et de matériels pédagogiques.

L'élaboration du curriculum d'établissement est le terrain concret où peut se mesurer la capacité réelle de chaque établissement autonome à se projeter véritablement. Le POF est en effet «le document constitutif de l'identité culturelle de chaque établissement et de sa capacité à se projeter».

Une deuxième étape décisive de la réforme est celle de la loi de 2003 $\left(n^{\circ} 53\right)$ : il n'est plus question de curriculum mais de "plans d'étude personnalisés ».

7. Le curriculum de chaque établissement, qui est défini aussi à travers une intégration de différents systèmes de formation, sur la base d'accords avec les régions et les collectivités locales, peut également tenir compte de projets et accords internationaux. 


\begin{tabular}{|c|c|}
\hline Au plan opérationnel, le ministère définit : & Au plan opérationnel, l'établissement définit : \\
\hline $\begin{array}{l}\text { 1. les objectifs généraux ; } \\
\text { 2. l'horaire annuel (somme de la partie arrêtée } \\
\text { nationalement et de celle arrêtée au niveau } \\
\text { de l'établissement); } \\
\text { 3. les disciplines, les activités, les horaires annuels } \\
\text { correspondants ; } \\
\text { 4. les objectifs spécifiques d'apprentissage } \\
\text { (les curricula disciplinaires); } \\
\text { 5. les obligations de service des enseignants ; } \\
\text { 6. les indications générales relatives à l'évaluation } \\
\text { des élèves, les modèles pour la certification } \\
\text { des compétences; } \\
\text { 7. les critères généraux pouvant définir } \\
\text { les parcours de formation dans le domaine } \\
\text { de la formation permanente. }\end{array}$ & $\begin{array}{l}\text { 1. les disciplines, les horaires et les objectifs } \\
\text { qui correspondent à la partie dont il a la maîtrise } \\
\text { (son « quota »); } \\
\text { 2. les modalités de mise en ouvre des actions } \\
\text { pédagogiques dans le cadre de la flexibilité prévue } \\
\text { (modules, classes ouvertes, unités d'enseignement, } \\
\text { parcours individualisés); } \\
\text { 3. le développement de l'offre de formation } \\
\text { en cohérence avec la finalité des différents cursus. }\end{array}$ \\
\hline
\end{tabular}

Quelle différence ? Tandis que le projet curriculaire se réclame de l'uniformité de l'offre, le plan d'études personnalisé est plus tourné vers la situation de chaque élève. La loi, tout en confirmant les prérogatives de chaque école, dispose que les plans d'études personnalisés devront certes contenir un noyau fondamental homogène au plan national, mais prévoit aussi qu'à côté de cette partie nationale, une partie devra être ultérieurement réservée aux régions, sur des sujets qui les intéressent particulièrement, et en liaison avec les réalités locales.

Depuis 2000, en parallèle à l'affirmation générale, dans le pays, d'une forte tendance vers l'autonomie et la participation, l'approche centralisée de l'État et du ministère a été limitée, au bénéfice de l'accroissement du rôle de l'autonomie des établissements scolaires, mais aussi des régions et des communes. En conséquence, les curricula ont été élaborés à partir de textes spécifiques conçus par des commissions d'experts et à la suite de consultations avec les partenaires sociaux, avec les associations professionnelles d'enseignants, les associations scientifiques et culturelles, les représentants des parents et des élèves ${ }^{8}$.

Dans cette perspective, le curriculum se présente pour l'école comme une sorte de mosaïque dont seulement une partie est prescriptive, le curriculum national ${ }^{9}$ émanant du ministère : il s'agit des connaissances et compétences de base, obligatoires quel que soit le cycle d'études visé, telles qu'elles figurent dans les Indications nationales.

8. En 2006, le ministre de l'Éducation Fioroni (centre-gauche) a fait appel à Edgar Morin pour valider les Instructions nationales élaborées pour le curriculum de l'école de base. En 2007, il a créé une commission de recherche bipartisane chargée de la réforme des curricula des instituts techniques et professionnels. En 2008, le nouveau ministre Gelmini (gouvernement Berlusconi de centre-droit) a confirmé cette commission et les axes stratégiques qui avaient été arrêtés.

9. Dans trois régions italiennes à statut spécial (Val d’Aoste, province de Trente et province de Bolzano), l'autonomie curriculaire est beaucoup plus forte le seul élément qui n'en relève pas est l'examen terminal d’État. 


\section{TYPOLOGIE ET FORMES DE CURRICULA}

Les tendances les plus récentes des réformes curriculaires en Italie peuvent être résumées de la façon suivante:

\section{Direction $n^{\circ} 1$ : la conception verticale du curriculum}

Il s'agit d'un effort pour donner un caractère cohérent et progressif aux enseignements dispensés dans le premier cycle de l'éducation (correspondant aux âges 3-16 ans), dépassant la séparation traditionnelle entre le vieil enseignement primaire et celui délivré dans les traditionnelles «écoles moyennes ». Les instructions nationales de 2007 introduisent un ensemble d'objectifs, en termes de compétences et redessinent les parcours selon différents "champs d'expérience » pour l'école maternelle, ainsi que par "aires d'apprentissage ». Ces dernières se définissent par rapport à chaque discipline aussi bien qu'en tant que savoirs transversaux.

L'école maternelle définit cinq "champs d'expérience»:

- soi et l'autre (les grandes questions, le sens moral, le vivre ensemble);

- corps et mouvement (identité, autonomie, santé) ;

- langages, créativité, expression (gestuelle, art, musique, multimédias);

- discours et paroles (communication, langue, culture);

- connaissance du monde (ordre, espace, temps, nature).

École élémentaire et école moyenne délimitent trois aires multidisciplinaires :

- l'aire linguistique-artistico-expressive (italien, langues européennes, musique, arts plastiques et image, corps, mouvement et sport) ;

- l'aire historico-géographique (les deux disciplines en question);

- l'aire mathématico-scientifique-technique (mathématiques, sciences naturelles et expérimentales, technologie).

\section{Direction $n^{\circ} 2$ : centration sur l'essentiel des contenus didactiques pour favoriser l'acquisition d'une base commune de compétences nécessaire à l'exercice de la citoyenneté}

Cette tendance concerne surtout la seconde partie de l'éducation (le secondaire supérieur) et apparaît principalement dans le récent décret sur l'obligation scolaire (décret ministériel $n^{\circ} 139$ du 22 août 2007) ainsi que dans les Instructions récentes pour les lycées et les instituts techniques et professionnels (approuvées en 2010).

Le décret sur l'instruction obligatoire prévoit en effet quatre " axes culturels » communs à tous les cursus (langages, mathématiques, sciences et technique, histoire/sciences humaines), axes renvoyant à vingt-quatre compétences culturelles essentielles liées de près aux huit compétences-clés pour l'exercice de la citoyenneté définies au niveau européen.

L'individualisation des savoirs et des compétences, telle qu'elle est définie dans les nouveaux textes officiels sur l'obligation scolaire, "garantit l'équivalence de tous les parcours en termes de formation, dans le respect de l'identité de 
l'offre formative et des objectifs propres qui caractérisent les cursus des différents niveaux d'études, voies et filières ». Ce qui, en particulier pendant les deux années de fin d'instruction obligatoire, est garanti à tous, du lycée à la formation professionnelle, est la maîtrise des compétences-clés, et non celle des disciplines.

Cela conduit à privilégier l'individualisation des « structures profondes » et des "paradigmes» des matières d'enseignement, ou encore de ces " concepts clés " grâce auxquels il est possible d'extraire et de maîtriser les différentes combinaisons de savoirs qui sont nécessaires à l'acquisition des compétences.

\section{Direction $n^{\circ} 3$ : introduction de standards de contenus et de dispositifs de certification des compétences}

Les programmes traditionnels étaient jugés surdimensionnés par rapport aux possibilités réelles de l'école, et regardés davantage comme une « carte culturelle » du curriculum que comme un véritable parcours conduisant à la maîtrise des compétences. D'où la difficulté d'introduire un système national d'évaluation des apprentissages et des standards réellement mesurables.

Or depuis trois ou quatre ans s'est produite une évolution qui a conduit à :

- introduire (en 2010-2011) un dispositif de certification des compétences à la fin de l'école obligatoire (16 ans);

- consolider et généraliser le Sistema Nazionale di Valutazione (SNV) coordonné par l'Invalsi, qui consiste à soumettre les élèves de toutes les écoles du cycle obligatoire à des épreuves standardisées $\left(3^{\mathrm{e}}\right.$ et $5^{\mathrm{e}}$ année de primaire et $3^{\mathrm{e}}$ année d'école moyenne) ;

- élaborer les premiers standards de compétences (à partir de ce qui s'est fait en formation des adultes et dans le domaine de la formation professionnelle).

Les réductions drastiques du budget de l'éducation n'ont en revanche pas permis d'augmenter la part des enseignements optionnels proposés aux élèves : les nouveaux curricula prévoient bien de telles possibilités sur le papier, dans un cadre flexible, mais ces possibilités entrent en collision avec les réductions touchant les personnels et les moyens en heures. C'est seulement dans certaines régions autonomes qu'il est possible d'observer, à ce stade, des innovations qui comportent l'introduction d'une portion du curriculum flexible pour répondre à la personnalisation des parcours.

\section{CURRICUlum ET POLITIQUe CURRICULAIRE}

\section{Curriculum et pratiques pédagogiques}

L'étude du curriculum se joue, donc, entre deux pôles : d'un côté, parler de curriculum signifie parler d'un optimum pédagogique, déterminé par des choix de politique éducative, à la fois objectifs de formation à atteindre, contenus 
disciplinaires à transmettre, stratégies didactiques à mettre en œuvre, instruments de contrôle et critères d'évaluation. D'un autre côté, parler de curriculum veut dire parler des résultats effectivement obtenus à la suite d'actions pédagogiques, intentionnelles ou de façon spontanée.

La tradition italienne de "programmes » décrétés d'en haut était une entreprise d'apparence "titanesque ", qui conduisait à tracer sur le papier des objectifs aussi élevés que nombreux, mais sans qu'on retrouve chez les élèves des niveaux effectifs de maîtrise en rapport avec de tels objectifs.

Les nouvelles perspectives curriculaires semblent aller dans une direction différente et amener avec elles, de façon inévitable, de nouvelles réflexions sur l'évaluation. D'un côté, on assiste bien à une orientation plus marquée vers la valorisation des performances et la diffusion de preuves bien construites, mais d'un autre, on est confronté à une richesse curriculaire plus attentive à la qualité et, en conséquence, plus complexe et difficile à " contrôler ».

Le défi de l'évaluation semble du reste à peine lancé : tous les rapprochements opérés par l'Invalsi entre les résultats des évaluations faites par les enseignants et celle venant d'enquêtes internationales (comme PISA, TIMSS, PIRLS) montrent encore un écart considérable entre la subjectivité des professeurs italiens, quand ils évaluent les performances, et l' « objectivité » que permettent les comparaisons internationales.

\section{Le curriculum et le soutien aux écoles et aux enseignants}

Pour ce qui concerne les personnels la mise en place des nouveaux curricula rend indispensable un investissement important pour soutenir la formation et l'innovation.

Il est en effet évident que, par rapport à l'époque des programmes nationaux, la logique de ces curricula crée une situation différente pour tout ce qui concerne d'abord le métier des professeurs comme des cadres de l'éducation. Le programme prescrit une liste d'objectifs et de contenus définie centralement et qui fait abstraction de toute référence aux réalités locales : le professeur doit se référer à cette liste et la mettre en pratique dans son enseignement, en faisant place aux réalités sociales dans lesquelles l'école est insérée, à sa culture propre, aux exigences spécifiques nées de l'écoute des besoins des élèves, de la réponse aux demandes et attentes des familles et de l'environnement local. Si, dans le cadre des programmes, on demandait aux enseignants d'être de bons exécutants de prescriptions élaborées ailleurs, dans le cadre du curriculum, il leur est demandé d'être des co-élaborateurs, responsables de leurs choix. La communauté professionnelle est appelée à assumer au fond la responsabilité de ce qu'elle fait, dans un cadre qui reconnaît pleinement la liberté culturelle de chacun, avec une dimension collective de collaboration, de négociation des choix, de partage d'une idée particulière d'école. 
Le processus de construction du curriculum ne se conclut pas une fois pour toutes, mais prend la forme d'une recherche continue, grâce à l'action des professeurs, en tant que professionnels réflexifs impliqués dans une activité constante d'analyse et de reconsidération de leurs pratiques ${ }^{10}$. Ce point requiert en outre une forte rénovation de l'organisation pédagogique dans l'établissement pour qu'elle soit en phase avec la mise en place de nouveaux environnements d'apprentissages.

Devant ce scénario, les politiques éducatives ont dû intervenir :

- pour soutenir les projets provenant de l'initiative d'une école individuelle ou d'un réseau d'écoles, dans le sens du développement du curriculum local et/ou de l'innovation pédagogique ;

- pour former des plans nationaux et régionaux de réactualisation de la formation des personnels, dans le domaine des nouvelles méthodes pédagogiques ainsi que des technologies;

- pour pousser des plans d'équipement des écoles, en matière de laboratoires et d'outils pédagogiques (en particulier avec les tableaux interactifs), et d'accords avec les éditeurs scolaires.

Hélas la réduction progressive des budgets de l'éducation décidée par les derniers gouvernements n'a aidé ni les écoles ni l'opinion publique à percevoir l'éducation comme terrain prioritaire d'investissement, et cela a même favorisé une attitude de passivité vis à vis du changement.

Les établissements scolaires ont répondu de façon très diverse, tantôt avec des comportements de type bureaucratique/défensif, tantôt (mais de façon encore minoritaire) en acceptant les défis de l'innovation dans le domaine des curricula, de la didactique et de l'organisation pédagogique.

Le thème de l'autonomie a été vécu par beaucoup au cours des dernières années d'abord comme quelque chose d'assez formel, de caractère juridique et imposé, amené et souhaité surtout par le monde extérieur à l'école (le marché, les collectivités locales, les institutions), avec l'objectif de mettre l'école en phase avec son temps, de la moderniser. En somme, c'est une comédie à deux personnages : d'un côté une société en transformation rapide, de l'autre l'école avec ses temps longs (qui la placent presque à la limite de l'obsolescence). Cette idée est toutefois trompeuse, en ce qu'elle ne prend pas en compte le fait que l'école requiert des temps longs et désintéressés, sa mission étant de contribuer à construire chez les élèves une identité, une mémoire, des savoirs durables.

L'autonomie, en effet, n'est pas la consécration d'une culture de type entrepreneurial, en quête de la recherche de l'efficacité procédurale, mais avant tout un processus culturel et professionnel, qui vise la possibilité de mettre en place un «bon » enseignement, apprécié en tant que tel par la société.

10. Une des propositions les plus efficaces en matière de formation continue s'est avérée d'activités de rechercheaction par petits groupes stables d'enseignants de la même école, avec une durée prévue sur quelques années. 
Ce type d'école demande aussi qu'émerge un leadership « réparti », c'està-dire que soit reconnu, dans chaque école, un groupe qui stimule les autres (qui fasse équipe avec le chef d'établissement), qui sache toutefois développer aussi la participation et la professionnalité de chacun, son sens d'appartenance à l'établissement autonome et sa volonté de contribuer à la qualité de l'école.

Dans la phase pionnière a souvent prévalu, en Italie, l'idée qu'on pouvait réaliser l'autonomie en rajoutant quelques activités au curriculum (élargissement et enrichissement de l'offre formative), peut-être en organisant des parcours novateurs, en tous cas seulement des choses facultatives.

Or l'autonomie pédagogique et didactique ne signifie pas nécessairement l'augmentation de l'offre par rajouts, mais plutôt se réinterroger sur le curriculum central, sur les objectifs de formation dans l'établissement, sur les compétences à développer, sur les objectifs de formation qu'on veut atteindre à travers les enseignements disciplinaires.

Stenhouse a bien dit que le curriculum est un projet ouvert de recherche, un « essai pour communiquer les principes et les principaux traits d'une proposition éducative de façon qu'elle reste ouverte à toute révision critique ».

Mais, tandis que les écoles italiennes du premier cycle ont délibérément faite leur l'idée du curriculum comme activité formatrice à activer à partir des situations réelles, l'école secondaire supérieure est en revanche demeurée solidement ancrée dans une perspective de focalisation sur les contenus, au delà de toutes les déclarations de principe, avec l'idée que le curriculum est conçu comme un bloc de contenus qu'on peut parfaitement attribuer aux différentes disciplines, et qui doivent de quelque façon que ce soit être acquis par les élèves.

Parler de curriculum signifie au contraire la nécessité de définir les bornes d'un espace exactement opposé : capacité à projeter des actions au niveau local, volonté de centrer les actions sur des objectifs de formation partagés, prise en compte de la complexité.

Le «fédéralisme en matière scolaire »- qui a été récemment introduit dans la norme italienne - pourrait constituer un moyen de réduire les différences qui existent au niveau local, mais il pourrait aussi les accentuer si devait prévaloir un «fédéralisme par abandon » de la part des régions les plus développées.

Les «périphéries » semblent prendre leur revanche sur le «centre », en matière d'éducation aussi, mais on peut être préoccupé par la question de l'unité du système éducatif au plan national et par la garantie qui peut exister quant au maintien de la cohésion sociale et de l'équité. Toutefois la reconnaissance constitutionnelle de l'autonomie des écoles peut représenter une garantie de stabilité et de solidité d'un système fondé sur les établissements : les prérogatives de chaque école en matière culturelle, pédagogique et d'organisation ne pourront pas être foulées aux pieds par les pouvoirs locaux, de même que l'État devra limiter ses interventions aux normes globales, aux niveaux essentiels des services et aux principes généraux. Le défi semble à peine lancé. 